Proceedings of the International School and Conference on Optics and Optical Materials, ISCOM07, Belgrade, Serbia, September 3-7, 2007

\title{
Realisation of Transparency below the One-Photon Absorption Level for a Coupling Laser Driving a Lambda System under EIT Conditions
}

\author{
L. Spani Molella, R.-H. Rinkleff and K. Danzmann
}

Albert-Einstein-Institut, Max-Planck-Institut für Gravitationsphysik and Institut für Gravitationsphysik

Gottfried Wilhelm Leibniz Universität Hannover

Callinstr. 38, 30167 Hannover, Germany

The concept of parametric spectra in electromagnetically induced transparency is clarified. In this context, the possibility for a coupling laser to reach absorption levels below the one-photon absorption is experimentally shown. In this sense, the first results of electromagnetically induced transparency for a coupling laser in a coherent-population-trapping dominated system are presented.

PACS numbers: 42.50.Gy, 42.62.Fi

\section{Introduction}

The physics of electromagnetically induced transparency (EIT) is well understood [1, 2] both from the theoretical and experimental point of view. In a $\Lambda$ system, for example, it is known that a probe laser which probes an atomic two-level transition is affected by transparency at the resonance conditions if the upper level is shared with a second transition coupled by a coupling laser.

However, in describing the physics of EIT systems, the greatest number of discussions concern the probe laser, which indeed experiences an almost complete transparency in correspondence with the two-photon resonance conditions. Still, when it comes to the properties of the coupling laser, i.e. the laser which drives the associated fixed-frequency two-level transition, the results are much limited in number and of not so obvious interpretation (one positive exception is constituted by $[3])$.

In this publication, the authors want to clarify what has to be understood under electromagnetically induced transparency for the coupling laser and show 
how and why such spectra differ qualitatively from those of the probe laser. In this context, we will see the crucial role played by the residual coupling-laser absorption level when the probe laser is off resonant.

\section{Definitions and experimental setup}

The EIT measurements presented in this publication were conducted in one of the two $\Lambda$ level schemes available within the hyperfine structure of the $D_{2}$ transition in atomic caesium (Fig. 1).

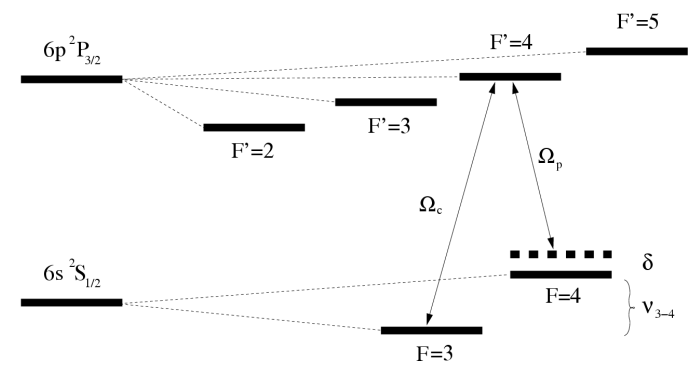

Fig. 1. Level structure of the $D_{2}$ line in caesium and $\Lambda$ system used throughout the experiment.

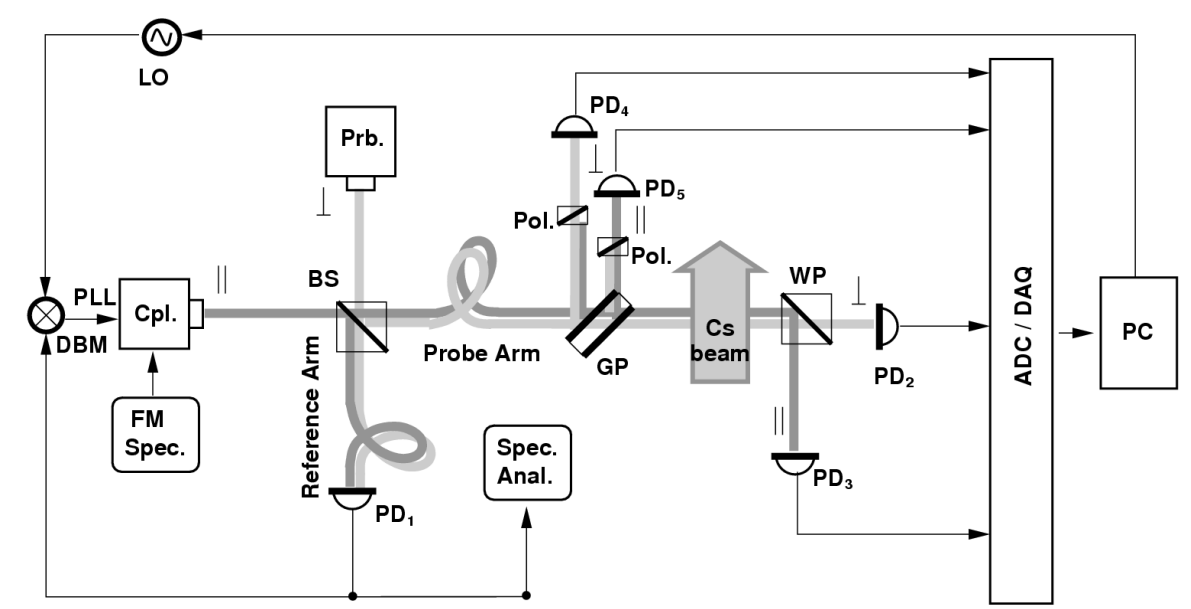

Fig. 2. Experimental setup: Cpl., Prb. — coupling and probe laser; Pol. — polariser; WP - Wollaston prism; GP — glass plate; Cs beam - caesium atomic beam; PD photodiode; LO — local oscillator; PLL — optical phase-locked loop; DBM — double balanced mixer; BS - power beam splitter; FM Spec. — frequency modulation spectroscopy; Spec. Anal. — spectrum analyser; ADC/DAQ - digital data acquisition card; PC — personal computer. 
The coupling and the probe laser driving and probing the respective transitions were arranged in a heterodyne interferometer. A sketch of the experimental setup is given in Fig. 2. The lasers were external-cavity-stabilised diode lasers. They were phase-locked to each other with a frequency difference $\nu=\nu_{3-4}+\delta$, where $\nu_{3-4}$ was the frequency of the hyperfine transition between the hyperfine levels of the ground state - i.e. roughly $9.192 \mathrm{GHz}$ - and $\delta$ the variable frequency difference used to sweep the probed transition. The caesium was prepared in form of an atomic beam propagating orthogonally to the direction of propagation of the lasers to minimise the Doppler broadening of the lines. The interaction area was shielded from magnetic influences through an active compensation of the local magnetic fields. For this reason, assuming the degeneracy in the Zeeman structure of the hyperfine levels, we neglected it in the further analysis of the system. The setup can be considered as a simplified version of the three-beam heterodyne interferometer described in [4], which was actually used to perform the measurements. However, because the measurements presented here did not need the use of a third laser, we omitted it in the description of the experiment. We refer to the cited publication for any further experimental detail.

\section{Experimental results}

Before presenting the experimental results, it is opportune to clarify a crucial point concerning the coupling-laser parametric absorption spectra (we call parametric spectra the coupling laser spectra as a function of the probe laser detuning from the two-photon resonance frequency). The off-resonant absorption level at the base of such an absorption curve corresponds to the coupling absorption level for an off-resonant probe laser. When the probe is off-resonant some coupling laser absorption is expected, because the coupling laser frequency is resonant with the frequency of the coupled two-level transition. For this reason, one can claim that the coupling spectra for the $F=3 \rightarrow F^{\prime}=4$ transition do not strictly show an enhanced transparency if the coupling-laser absorption at the EIT resonance does not reach at least the absorption level related to the pure one-photon transition. This observation can be applied for example to the $\Lambda$-system spectra published in $[5,6]$.

In the case of such $\Lambda$-systems we were indeed able to measure parametric absorption levels below the one-photon level, i.e. real transparency also for the coupling laser, as shown in Fig. 3. In a proper sense, these have to be considered the first observations of electromagnetically induced transparency measured in the spectra of the coupling laser, because only in this case a transparency is actually achieved. For completeness, the probe laser spectrum measured in association with the coupling parametric absorption spectrum is also shown.

To double-check the results we measured the spectra over a broad frequency interval, sweeping the probe detuning up to $100 \mathrm{MHz}$ off resonance. In this way we were sure to determine exactly the value of the coupling laser absorption coefficient 

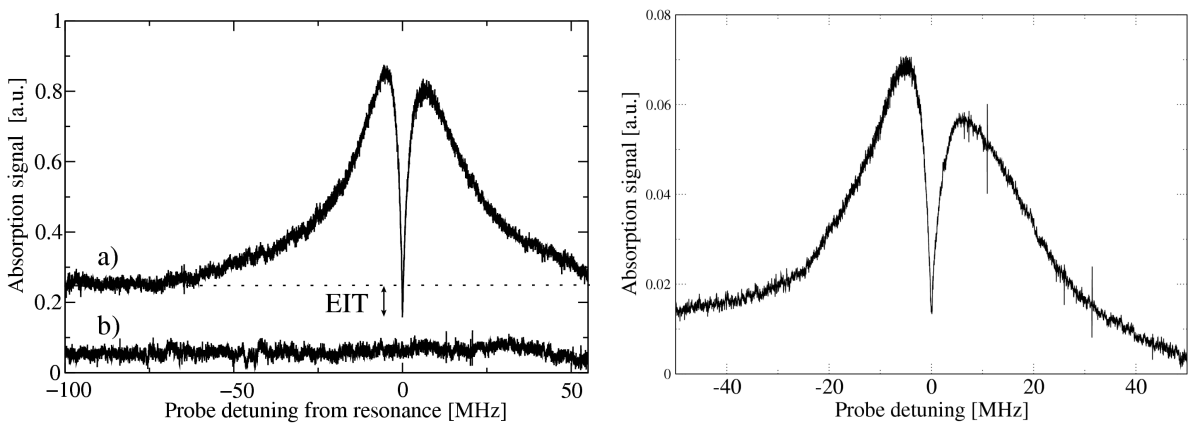

Fig. 3. (left) EIT signal measured in arbitrary unuts in the $\Lambda$ system of Fig. 1 showing how the absorption of the coupling laser can be reduced below the one-photon absorption level in case of a coherent interaction between the coupling and the probe laser. Curve $a$ ) shows the coupling parametric absorption, curve $b$ ) the signal observed at the photodiode in absence of the caesium beam under the same experimental conditions. The dotted curve represents the level of the base of the coupling absorption curve corresponding to the one-photon absorption. (right) Probe absorption signal accompanying the previous coupling laser parametric absorption.

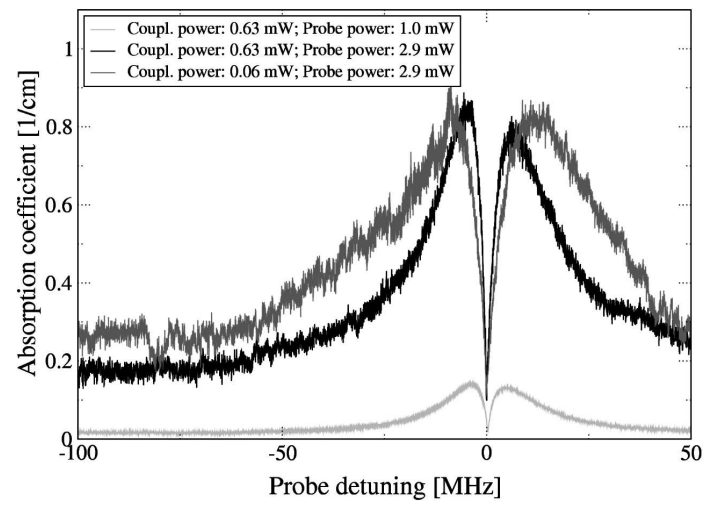

Fig. 4. EIT signal measured in the $\Lambda$ system of Fig. 1 showing the absorption of the coupling laser for three different probes to coupling laser power ratios.

at the base of the resonance curve, i.e. in the case of a one-photon transition induced by the coupling laser only.

It must be also noted that such an effect could not have been induced even through an imperfect preparation of the probe and coupling polarisations or by rotations in the polarisation induced by the atomic medium. Indeed, the probe-laser absorption level cannot be lower than the level corresponding to the base of its own absorption curve, where the probe laser is off-resonant and, as a consequence, not absorbed by the medium. Therefore, even in case of a non-exact separation of the probe and the coupling beam at the Wollaston prism, the falsely 
detected probe light on the photodiode sensing the coupling absorption could not give rise to the observed effect and no additional probe transparency could have been accidentally transferred to the coupling laser. Still, it is interesting to note that the coupling laser transparency was more pronounced in the experimental configuration corresponding to a weak coupling laser and a strong probe laser, as it can be seen in Fig. 4, and became more marked in case of high power ratios between probe and coupling laser. When the coupling intensity was stronger than the probe intensity the coupling spectra at the two-photon resonance reached only the absorption level of the base of the resonance curve, in agreement with published theoretical predictions (e.g. Fig. 4 in [3]).

\section{Conclusions}

With this publication we suggest a redefinition of what has to be understood as electromagnetically induced transparency in the parametric absorption spectra of the coupling laser (the absorption spectra as a function of the probe detuning): EIT for the coupling laser is present only if the induced transparency at the twophoton resonance is greater than the transparency level corresponding to the onephoton coupled transition. We presented measurements of coupling transparencies at the two-photon resonance, which lie below the one-photon absorption level and could be induced only by the coherent interaction between probe laser, coupling laser, and the atomic ensemble. They demonstrate that in a $\Lambda$ system it is indeed possible to induce a real transparency not only for the probe laser, but also for the coupling laser.

\section{Acknowledgments}

This project was supported by the Deutsche Forschungsgemeinschaft (German Research Foundation) through the Collaborative Research Centre SFB407.

\section{References}

[1] S.E. Harris, Phys. Today 50, 36 (1997).

[2] E. Arimondo, in: Progress in Optics, Ed E. Wolf, Vol. 35, Elsevier Sci., Amsterdam 1996, p. 257.

[3] C. Goren, A.D. Wilson-Gordon, M. Rosenbluh, H. Friedmann, Phys. Rev. A 69, 053818 (2004).

[4] L. Spani Molella, R.-H. Rinkleff, K. Danzmann, Spectrochim. Acta A 63, 987 (2006).

[5] M. Müller, F. Homann, R.-H. Rinkleff, A. Wicht, K. Danzmann, Phys. Rev. A 62, 060501(R) (2000).

[6] M. Müller, F. Homann, R.-H. Rinkleff, A. Wicht, K. Danzmann, Phys. Rev. A 64, 013803 (2001). 